\title{
Erratum: Hybrid Long-Distance Entanglement Distribution Protocol [Phys. Rev. Lett. 105, 160501 (2010).]
}

\author{
J. B. Brask, I. Rigas, E. S. Polzik, U. L. Andersen, and A. S. Sørensen \\ (Received 3 April 2012; published 23 May 2012)
}

DOI: 10.1103/PhysRevLett.108.219901

PACS numbers: 03.67.Bg, 03.67.Hk, 42.50.Ex, 99.10.Cd

In the course of recent work, we have discovered an error in the numerical simulations of the repeater. The error leads to a value of the rate which is too high.

A plot of the corrected rate is shown in Fig. 1 along with the rate from the published Letter for comparison. At a distance of $1000 \mathrm{~km}$, the corrected rate is lower by a factor of $\sim 95$, leading to a value of 0.001 rather than 0.1 pairs/minute as stated in the Letter. The protocol thus does not compare as favorably against discrete-variable repeater schemes as originally claimed. At $600 \mathrm{~km}$ we can compare against the schemes of the review [1]. We find that the corrected rate is not better than, but rather on par with, the rate of the best previous schemes that do not use multiplexing, when a realistic single-photon detector efficiency of $50 \%$ is assumed.

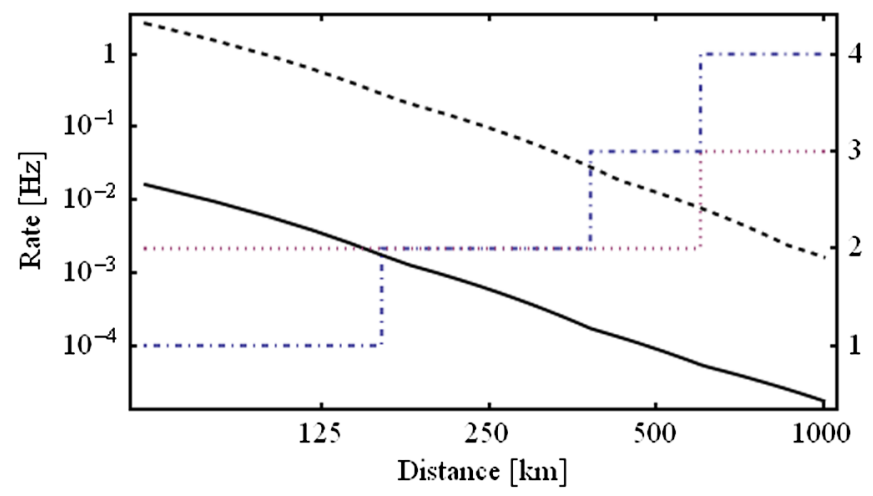

FIG. 1 (color online). Comparison of the corrected (solid black line) and the erroneous (dashed black line) rate. The optimal values of the number of generation steps $m$ (red dotted line), and connection steps $n$ (blue dash-dotted line) for the corrected rate are also indicated, with values given on the right-hand axis.

[1] N. Sangouard, C. Simon, H. de Riedmatten, and N. Gisin, Rev. Mod. Phys. 83, 33 (2011). 